\title{
Embolisation in the management of epistaxis in patients with hereditary haemorrhagic telangiectasia: a systematic review
}

\author{
Nikhil Shah ${ }^{1}$, Lawrence Nip ${ }^{1}$, Maha Khan² \& Katherine Conroy ${ }^{2}$ \\ 1. University College London \\ 2. North Western Deanery
}

\section{Introduction}

Hereditary haemorrhagic telangiectasia (HHT), also referred to as OslerWeber-Rendu disease, is an inherited disorder of the vasculature with consequent involvement and sequelae in a number of different organ systems. It is characterized by the appearance of telangiectasia, most prominently in the skin and mucosa of the respiratory and gastrointestinal tracts, and spontaneous bleeding from multiple sites. Epistaxis is a prominent feature of the condition, occurring multiple times per day in the most affected with potential life threatening consequences.

There remains much debate regarding the best treatment for these patients. As of now, no treatment offers a cure and there is lack of consensus regarding the most effective way to manage epistaxis in a patient with HHT.

\section{Aims \& Objectives}

The aim of this systematic review was to evaluate the efficacy of embolisation therapy in the management of epistaxis in patients with HHT. Five papers were selected for critical appraisal, with the key findings shown in the table below.

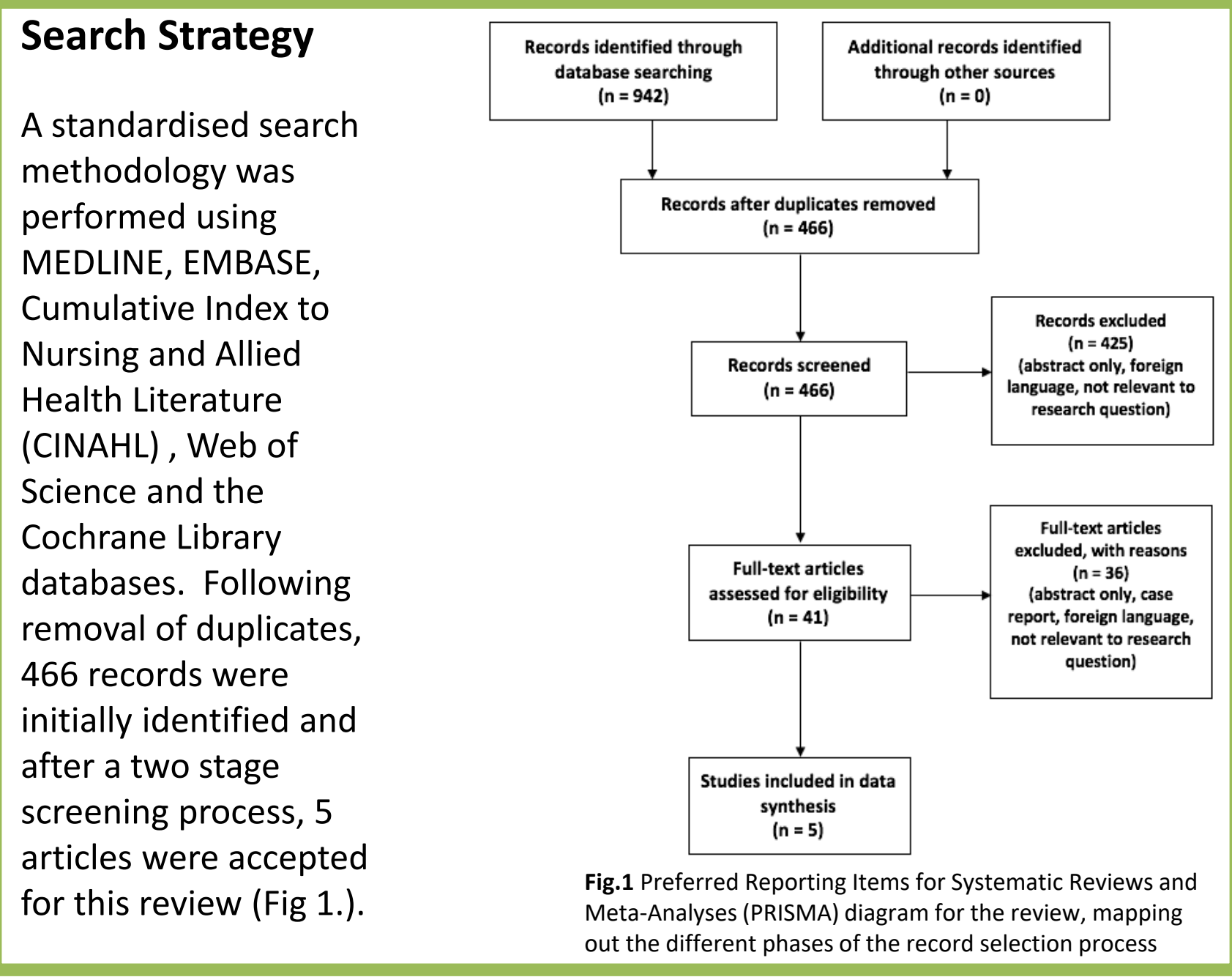

Comment

Kev results

. Epistaxis recurred following $13 / 15$ procedures on the 9 patients with HHT.

Aims: in HHT patients and patients with no pathology of the nasal cavity and identify the potential complications of embolization. following 12/15 procedures in patients with HHT 3. One serious complication was observed - necrosis of the nasa tip.

Frequency of epistaxis

Duration of epistaxis Number of major complications

Patients followed up by questionnaire and telephone interview.

S Braak et Patients with $H H T, n=12$ Case-control study Patients followed up by questionnaire and gathered from hospital records.

\section{Investigate and measure the results of} after 1 month and from 10.9 to $7.0(p=0.009)$ after 1 year. End points: Impact Factor (IF) defined as the daily frequency of epistaxis $x$ severity - Hb levels following therapy - Impact on lifestyle (IoL)

2. The mean $\mathrm{Hb}$ improved from $6.4 \mathrm{mmol} / \mathrm{l}$ to $7.2 \mathrm{mmol} / \mathrm{l}$ ( $\mathrm{p}$ 0.045 ) after 1 year.

loL after 1 year was positive following 12 procedures $(63 \%)$ and equal in the remaining $7(37 \%)(p=0.0001)$.

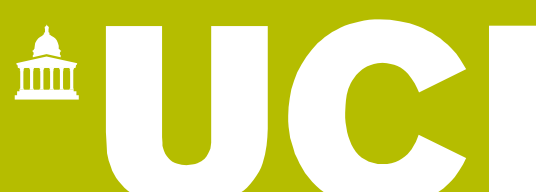

redis This study demonstrates good efficacy of embolization in with $\mathrm{H}$. with HHT. However, epistaxis was not completely stoppe and cannot therefore be seen as a long-term cure. Embolisation was shown to be relatively safe with only 1 documented major complication. The relatively small sample size and non RCT nature are limitations of this study. There may also be some recall bias and subjectivity due to the nature of the study design

\section{The result from this study confirms the effectiveness of} embolization in reducing the frequency and severity of epistaxis in patients with HHT. However there may be some recall bias and subjectivity due to the nature of the study are also limitations of this study. 22 patients ( 13 male, 9 female) treated with embolization divided into 2 groups:

Layton et al., 2007 Am J Neuroradiol

Patients with $\mathrm{HHT}, \mathrm{n}=12$

Patients without $\mathrm{HHT}, \mathrm{n}=10$

Compare outcomes following endovascular

embolization for epistaxis in patients with and without $\mathrm{HHT}$.

End points:

Patient records and procedure notes were - Angiographic findings reviewed retro

Requirement for multiple embolisations

Treatment efficacy Complication rates
1. Angiographic abnormalities were noted in 11 patients with $\mathrm{HHT}$ This paper demonstrates the efficacy of embolisation in $(83 \%)$ compared to 3 patients without $(30 \%)(p=0.0062)$. $50 \%$ of patients with $\mathrm{HHT}$ required repeated embolization, compared to $10 \%$ of patients without $\mathrm{HHT}(\mathrm{p}=0.0743)$.

3. Embolisation alone was able to control epistaxis in $50 \%$ of patients with $\mathrm{HHT}$, compared to $90 \%$ of patients with idiopathic epistaxis in these patients.

2/12 (17\%) HHT patients continued to have significant symptoms despite embolisation and surgery.
Limitations relate to the rarity of $\mathrm{HHT}$ and include smal sample size and the retrospective nature of the study.

\section{Strach et al., 2011. \\ Radiol \\ Case-control study \\ 48 patients presenting with intractable
epistaxis requiring embolisation divided epistaxis requirin
into 3 groups: \\ Patients with $\mathrm{HHT}, \mathrm{n}=5$ Idiopathic epistaxis, $n=31$ Traumatic/iatrogenic epistaxis, $n=12$ \\ Patient records and procedure notes were reviewed retrospectively. \\ Trojanowski et al., 14 patients with HHT (8 male, 6 female) 2011 Acta Radiologica Case-control study \\ admitted for endovascular management of epistaxis.}

Follow up over 24 month period - patients asked to report symptoms.

\section{$\begin{array}{ll}\text { Aims: } & \begin{array}{l}\text { 1. } 3 / 5 \text { patients with HHT required } 2 \text { embolisations. These patients } \\ \text { Evaluate outcomes following embolisation for life }\end{array}\end{array}$ experienced a reduction in fre \\ Severe complications were noted following 2/53 embolisations necrosis of the nasal tip and transient left hemiplegia lasting 4 days. Clinically indicated repeat embolisation was declined in $2 / 5$ Continuing epistaxis Reduced epistaxis frequency and duratio Cessation of epistaxis patients.} \section{Reduced frequency and duration of epistaxis wa
patients with HHT willing to undergo multiple embolisations. However, cessation of However, the sample size used limits any conclusions that an be drawn from the study.}

1. Immediate 6/14 (43\%) patients had recurer

enticacy of endovascular the risk of complications.

End points:

Initial control of acute bleeding Rate of recurrent nasal bleedin

Frequency of epistaxis

Severity of epistaxis

Number of complications
10 patients noted reduced frequency of epistaxis 24 months post-embolisation. 11 patients noted reduced severity of epistaxis. 2 patients were unavailable for follow up. 4. No major complications were noted.
Epistaxis offers a relatively low risk intervention with a high success rate in controlling acute bleeding, making it an intractable epistaxis.

Furthermore, it can significantly improve quality of life in patients with $\mathrm{HHT}$, in whom epistaxis can be a daily bleeding. occurrence, by reducing both frequency and severity of

\section{Conclusion}

Our systematic review has shown that embolisation is a safe and effective means of reducing the frequency and duration of epistaxis in patients with HHT. Complications are few and some authors document a significant improvement in quality of life. However, embolisation alone does not represent a long-term cure with some form of relapse occuring in the majority of patients. Nonetheless, we recommend that embolisation is a valid treatment option and clinicians may continue to opt for it in light of the evidence in favour of it. Further large scale studies with level I evidence are needed to confirm our findings and would be useful for clarification. In its current form, embolisation is not a definitive treatment option but selected patients with recurrent severe epistaxis may benefit.

1. Andersen, P., Kjeldsen, A. and Nepper-Rasmussen, J. (2005). Selective embolization in the treatment of intractable epistaxis. Acta Oto-Laryngologica, 125(3), pp.293-297.

. Braak S., de Witt C., Disch F. et al. (2009). Percutaneous embolization on hereditary hemorrhagic telangiectasia patients with severe epistaxis. Rhinology. 47(2), pp. 166-171.

3. Layton KF. (2007) Endovascular treatment of epistaxis in patients with hereditary hemorrhagic telangiectasia. American Journal of Neuroradiology. 28(5), pp. 885-888.

4. Strach K., Andreas S, Wilhelm K, et al. (2011). Endovascular treatment of epistaxis: indications, management, and outcome. Cardiovascular and International Radiology. 34(6), pp. 1190-1198 